\title{
Matrix-producing Breast Carcinoma: A Rare Subtype of Metaplastic Breast Carcinoma
}

Nektarios Koufopoulos ${ }^{1}$, Stefania Kokkali ${ }^{2}$, Foteini Antoniadou ${ }^{3}$, Dionysios T. Dimas ${ }^{4}$, Ioannis L. Missitzis 4

1. Pathology, Attikon University Hospital, Medical School of Athens, Athens, GRC 2. Oncology, Saint Savvas Cancer Hospital, Athens, GRC 3. Pathology, Saint Savvas Cancer Hospital, Athens, GRC 4. Surgery, Saint Savvas Cancer Hospital, Athens, GRC

Corresponding author: Nektarios Koufopoulos, koufonektar@yahoo.com

\begin{abstract}
Matrix-producing carcinoma (MPC) is a rare subtype of metaplastic breast carcinoma (MBC) that was first described in 1989 by Wargotz and Norris. It accounts for less than $1 \%$ of breast carcinomas and has distinctive clinical, morphological, and immunohistochemical features. Histologically it consists of invasive carcinoma of no special type with transition to cartilaginous or osseous matrix without a spindle cell component. Data on this entity are limited with the literature consisting mostly of case reports and a small number of case series.
\end{abstract}

We report a case of matrix-producing breast carcinoma, with excellent clinical outcome. We also discuss the histogenesis, imaging, histological, and immunohistochemical characteristics, treatment, and focus on the differential diagnosis of this rare tumor.

Received 06/09/2019

Review began 06/12/2019

Review ended 07/10/2019

Published 07/22/2019

๑) Copyright 2019

Koufopoulos et al. This is an open access article distributed under the terms of the Creative Commons Attribution License CC-BY 3.0., which permits unrestricted use, distribution, and reproduction in any medium, provided the original author and source are credited.
Categories: Pathology, General Surgery, Oncology

Keywords: matrix producing carcinoma, metaplastic breast carcinoma, local recurrence, distant metastasis, breast tumor

\section{Introduction}

Metaplastic breast carcinoma (MBC) is a heterogeneous group of malignant epithelial tumors that undergoes metaplasia into mesenchymal or squamous differentiation. This group currently includes fibromatosis-like metaplastic carcinoma, low-grade adenosquamous carcinoma, spindle cell carcinoma, carcinoma with mesenchymal differentiation, and squamous cell carcinoma. They are very rare accounting for less than $1 \%$ of breast carcinomas. Each subtype has a unique morphology and different prognosis. Matrix-producing carcinoma (MPC) is a rare subtype of MBC. It is defined as a carcinoma with a direct transition from invasive carcinoma no special type to cartilage or osseous component lacking an intervening spindle cell component [1]. Because of rarity, the literature on MPC is limited and consists mostly of case reports and a few case series.

We present a case of MPC with excellent clinical outcome and focus on the differential diagnosis. We also discuss the histogenesis, clinical and imaging findings, histological and immunohistochemical profile, treatment, and prognosis of this rare entity.

\section{Case Presentation}

A 65-year-old female patient with no previous history was admitted to the surgery department due to a palpable mass of the upper-outer quadrant of the left breast. A Breast Imaging Reporting and Data System 5 lesion measuring $23 \mathrm{~mm}$ in its greatest diameter were revealed on digital mammography. Frozen section was positive, while the sentinel lymph node biopsy was negative for malignancy. A mastectomy was performed (Poster presentation: Koufopoulos N, Pigadioti E, Dimas D, Tsouma E, Misitzis I, Khaldi L. Matrix Producing Breast Carcinoma. Report of a Case. 30th European Congress of Pathology; September 8-12, 2018). On gross examination, the tumor was relatively well-circumscribed, solid, and gray-white in color. On microscopic examination, the periphery of the tumor was more cellular with gradually diminishing cellularity towards the central area (Figure $1 \mathrm{~A}$ ). It consisted of solid areas, nests, tubular structures, as well as isolated single cells embedded in an extracellular chondromyxoid matrix (Figures $1 B-1 D$ ). The matrix was diffuse accounting for $50 \%$ of the tumor area. Tumor cells had enlarged nuclei with distinct nucleoli (Figure $1 E$ ). The matrix was high-grade according to the criteria set by Downs-Kelly et al. (Figure $1 F$ ) [2]. Focal areas of necrosis were present. An intervening spindle cell component, angiolymphatic invasion, or peripheral lymphocytic infiltrations were not identified. 


\section{Cureus}
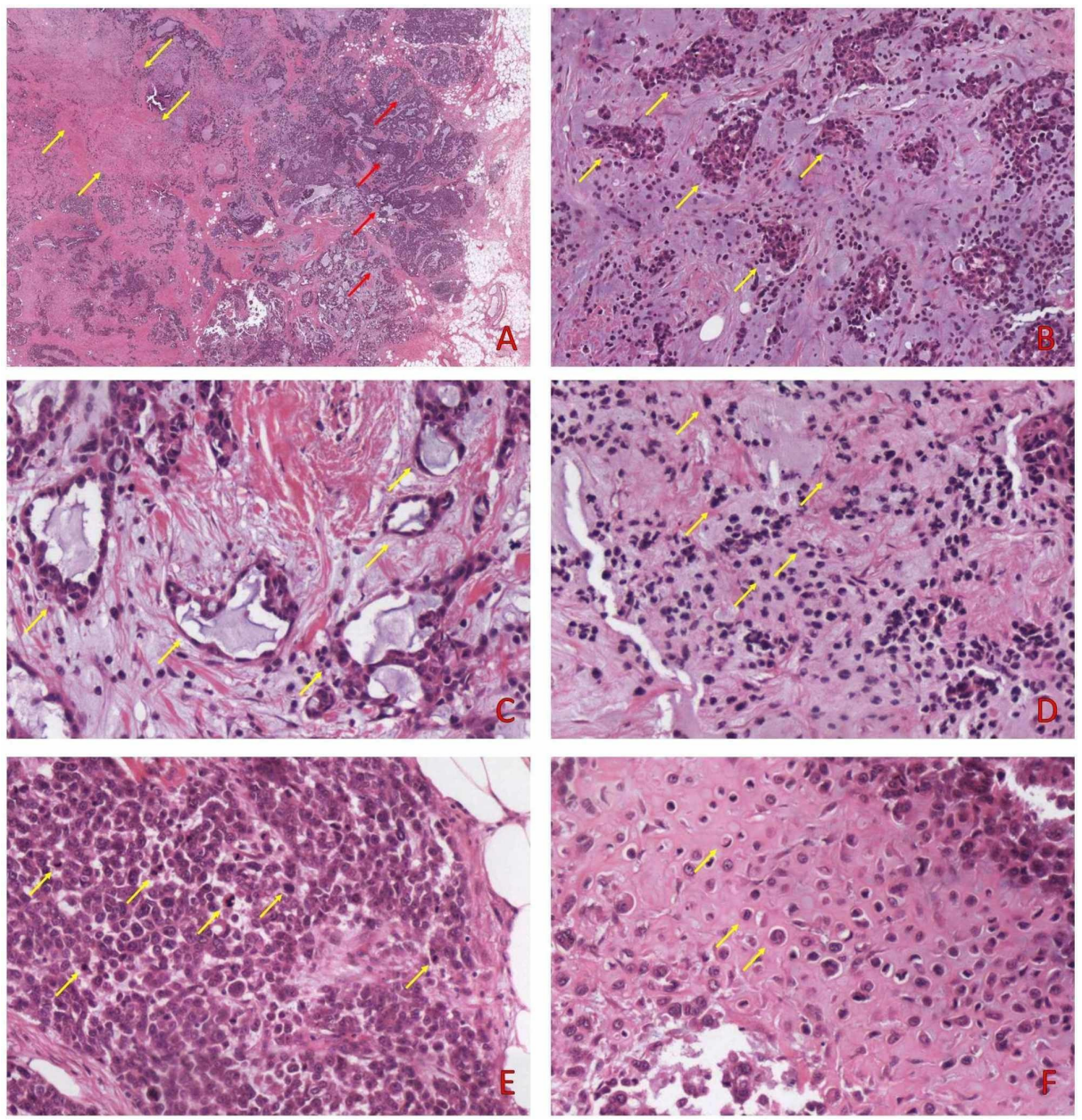

FIGURE 1: (A) On low power examination, MPC is more cellular peripherally (red arrows) with diminishing cellularity towards the center (yellow arrows); H\&E x10, (B) nests, (C) tubular structures and (D) isolated tumor cells embedded in an extracellular myxoid matrix; H\&E $x 200,(E)$ on high power examination, tumor cells were pleomorphic, had enlarged nuclei with distinct nucleoli. Several mitoses were identified; H\&E x200, (F) the matrix cells were hyperchromatic, had irregular nuclear contour, and a distinct nucleolus was visible; H\&E X200

H\&E: hematoxylin and eosin; MPC: matrix-producing carcinoma.

Immunohistochemical study was negative for Her-2, estrogen and progesterone receptors, cytokeratin 5/6, epidermal growth factor receptor (EGFR), P63, and S100. Proliferation index Ki67 stained 60\% of tumor nuclei.

The final diagnosis was MPC of the breast. The patient received adjuvant chemotherapy and is alive without evidence of recurrence or distant metastasis 38 months after surgery.

\section{Discussion}

$\mathrm{MBC}$ is a heterogeneous group of malignant epithelial tumors encompassing several different histologic subtypes, each one with a unique morphology and different clinical behavior. MPC is a rare subtype of MBC first reported in 1989 by Wargotz and Norris [1]. Its histogenesis is still unclear. Current literature suggests that MPC cells show epithelial features, either ductal or myoepithelial [3-4]. The neoplastic transformation of multipotent stem cells has been proposed in a few studies [5]. In some occasions, MPC has been associated with microglandular adenosis [6]. Schwartz et al. have recently reported a case of microglandular adenosis with evidence of molecular progression to MPC [7]. 
carcinomas of no special type [8]. On imaging studies (contrast-enhanced computed tomography and magnetic resonance imaging), MPC presents as a low-density mass with a peripheral ring structure enhancement [9-10]. On gross examination, the tumor is multinodular, well-circumscribed, hard in consistency, occasionally necrotic, and its color is grey-white. Microscopically, the tumor consists of malignant epithelial cells arranged in sheets, nests, cords, and tubular structures admixed with chondromyxoid or osseous matrix and sometimes with foci of hyaline cartilage [2]. Tumor cellularity is higher in the periphery and is gradually diminishing towards the center. Cellular atypia ranges from moderate to high-grade. Based on the nuclear characteristics (contour, pleomorphism, hyperchromasia), and whether visible nucleoli are present, the matrix has been classified as low-grade versus high-grade. Immunohistochemically, tumor cells usually express cytokeratin AE1/AE3, EGFR, and S100. They have almost always a triple-negative (ER, PR, and HER-2) immunophenotype.

MPC diagnosis is only possible in surgical specimens. Extensive sampling is required.

The differential diagnosis includes central acellular carcinoma (CAC) [11], primary or secondary chondrosarcoma, malignant phyllodes tumor with heterologous (chondorsarcomatous) differentiation [12], carcinoma ex-pleomorphic adenoma of the breast [13], and invasive lobular carcinoma with extracellular mucin production [14]. CAC and MPC both have a central acellular zone with a ring-like appearance. Their differences are that CAC lacks the chondromyxoid matrix of MPC and that there is a gradual decrease of neoplastic cells toward the central matrix in the MPC, whereas the transition between the cancerous and acellular zones in CAC is abrupt [15]. Proof of epithelial differentiation either morphologically or by immunochemistry excludes chondrosarcoma and malignant phyllodes tumor. Carcinoma ex-pleomorphic adenoma of the breast is composed of two components, one histologically similar to pleomorphic adenoma of the salivary gland and a malignant one resembling MPC [13]. In MPC the benign component is absent. Invasive lobular carcinoma with extracellular mucin production may also be included in the differential diagnosis since its mucinous component (single cells, clusters or cribriform structures floating in pools of mucin) may be confused with the matrix of MPC. However, the former shows non-mucinous areas of classic or solid type of invasive lobular carcinoma, signet ring cells, expression of estrogen receptors, and lack of Ecadherin expression [14].

MBC is usually treated aggressively due to the high stage at presentation [1]. Optimal treatment for MPC has not yet been defined since data are limited because of its rarity. Targeted gene therapy seems to play a role following genetic profiling in recent clinical trials [16].

Regarding prognosis, the literature has controversial results with reports claiming that MPC has a similar $[1,3]$ or a more aggressive clinical behavior than invasive breast carcinoma of no special type [2]. Compared with other types of MBC, it has a better prognosis [1]. Large tumor size (more than $5 \mathrm{~cm}$ ), lymph node infiltration, and high-grade matrix are associated with a worse outcome [5]. High-grade matrix is not related to tumor recurrence. Tumors with a significant amount of matrix ( $40 \%$ or more) seem to have a better prognosis [2].

In our case, we believe that the small tumor size, lack of lymph node infiltration, and the high percentage of the matrix may explain the lack of local recurrence or distant metastasis, despite the high-grade matrix.

\section{Conclusions}

MPC is a rare subtype of MBC with unique morphology, imaging features, and a triple-negative immunophenotype. Accurate diagnosis is impossible in core needle biopsy specimens and requires a surgical sample. It is usually treated with surgery, followed by adjuvant therapy. However, due to its rarity, optimal treatment has not yet been defined. Its prognosis is better compared to other types of metaplastic breast carcinoma.

\section{Additional Information \\ Disclosures}

Human subjects: Consent was obtained by all participants in this study. Conflicts of interest: In compliance with the ICMJE uniform disclosure form, all authors declare the following: Payment/services info: All authors have declared that no financial support was received from any organization for the submitted work. Financial relationships: All authors have declared that they have no financial relationships at present or within the previous three years with any organizations that might have an interest in the submitted work. Other relationships: All authors have declared that there are no other relationships or activities that could appear to have influenced the submitted work.

\section{References}

1. Wargotz ES, Norris HJ: Metaplastic carcinomas of the breast. I. Matrix-producing carcinoma . Hum Pathol. 1989, 20:628-635. 10.1016/0046-8177(89)90149-4

2. Downs-Kelly E, Nayeemuddin KM, Albarracin C, Wu Y, Hunt KK, Gilcrease MZ: Matrix-producing carcinoma of the breast: an aggressive subtype of metaplastic carcinoma. Am J Surg Pathol. 2009, 33:534-541. 
10.1097/PAS.0b013e31818ab26e

3. Rakha EA, Tan PH, Shaaban A, et al.: Do primary mammary osteosarcoma and chondrosarcoma exist? A review of a large multi-institutional series of malignant matrix-producing breast tumours. Breast. 2013, 22:13-18. 10.1016/j.breast.2012.09.010

4. Kusafuka K, Muramatsu K, Kasami M, et al.: Cartilaginous features in matrix-producing carcinoma of the breast: four cases report with histochemical and immunohistochemical analysis of matrix molecules. Mod Pathol. 2008, 21:1282-1292. 10.1038/modpathol.2008.120

5. Shruti S, Siraj F: Matrix-producing metaplastic breast carcinoma - a rare tumor with heterologous elements . Ger Med Sci. 2017, 15:17. 10.3205/000258

6. Liu LY, Sheng SH, Zhang ZY, Xu JH: A case of matrix-producing carcinoma of the breast with micoglandular adenosis and review of literature. Int J Clin Exp Pathol. 2015, 8:8568-8572.

7. Schwartz CJ, Dolgalev I, Yoon E, et al.: Microglandular adenosis is an advanced precursor breast lesion with evidence of molecular progression to matrix-producing metaplastic carcinoma. Hum Pathol. 2019, 85:65-71. 10.1016/j.humpath.2018.10.021

8. Bhosale SJ, Kshirsagar AY, Sulhyan SR, Sulhyan SR, Jagtap SV: Matrix-producing metaplastic breast carcinoma - a rare malignancy. Am J Case Rep. 2013, 14:213-215. 10.12659/AJCR.883958

9. Hirose T, Honda J, Bando Y, Sasa M, Hirose Y, Nagao T, Tangoku A: A case of matrix-producing carcinoma of the breast. World J Surg Oncol. 2008, 6:60. 10.1186/1477-7819-6-60

10. Inoue Y, Joden F, Yabuki K, et al.: A case of matrix-producing carcinoma of the breast [Article in Japanese] . J UOEH. 2017, 39:167-173. 10.7888/juoeh.39.167

11. Sasaki Y, Tsuda H, Ueda S, et al.: Histological differences between invasive ductal carcinoma with a large central acellular zone and matrix-producing carcinoma of the breast. Pathol Int. 2009, 59:390-394. 10.1111/j.1440-1827.2009.02382.X

12. Shui R, Bi R, Cheng Y, Lu H, Wang J, Yang W: Matrix-producing carcinoma of the breast in the Chinese population: a clinicopathological study of 13 cases. Pathol Int. 2011, 61:415-422. 10.1111/j.14401827.2011.02676.x

13. Hayes MM, Lesack D, Girardet C, Del Vecchio M, Eusebi V: Carcinoma ex-pleomorphic adenoma of the breast. Report of three cases suggesting a relationship to metaplastic carcinoma of matrix-producing type. Virchows Arch. 2005, 446:142-149. 10.1007/s00428-004-1137-7

14. Cserni G, Floris G, Koufopoulos N, et al.: Invasive lobular carcinoma with extracellular mucin production-a novel pattern of lobular carcinomas of the breast. Clinico-pathological description of eight cases. Virchows Arch. 2017, 471:3-12. 10.1007/s00428-017-2147-6

15. Yamaguchi R, Tanaka M, Mizushima Y, et al.: "High-grade" central acellular carcinoma and matrixproducing carcinoma of the breast: correlation between ultrasonographic findings and pathological features. Med Mol Morphol. 2011, 44:151-157. 10.1007/s00795-010-0522-3

16. Rossi L, Paglicci C, Caprio G, et al.: Matrix-producing carcinoma of the breast: a case report . Case Rep Oncol. 2013, 6:245-249. 10.1159/000351119 